\title{
Lettre de Sor Juana Inés de la Cruz à son confesseur, Mexique, 1681
}

\section{Marie-Cécile Benassy}

\section{(2) OpenEdition}

1 Journals

\section{Édition électronique}

URL : https://journals.openedition.org/clio/95

DOI : $10.4000 /$ clio.95

ISSN : 1777-5299

Éditeur

Belin

Édition imprimée

Date de publication : 1 avril 2002

Pagination : 181-190

ISBN : 2-85816-620-X

ISSN : $1252-7017$

Référence électronique

Marie-Cécile Benassy, «Lettre de Sor Juana Inés de la Cruz à son confesseur, Mexique, 1681 », Clio. Histoire, femmes et sociétés [En ligne], 15 | 2002, mis en ligne le 08 février 2005, consulté le 29 avril 2022. URL : http://journals.openedition.org/clio/95 ; DOI : https://doi.org/10.4000/clio.95 


\section{Lettre de Sor Juana Inés de la Cruz à son confesseur, Mexique, 1681}

Marie-Cécile BÉNASSY

Ce texte manuscrit non autographe du XVIII siècle n'est pas daté mais le contenu en situe la rédaction vers 1681 . Il était parfaitement inconnu jusqu'en 1980 quand le prélat mexicain Aureliano Tapia Méndez découvrit, dans sa ville de Monterrey, cette copie tardive d'un texte de sa compatriote Juana Ramírez de Asbaje (ou Asuaje), religieuse hiéronymite sous le nom de Sœur Jeanne Inès de la Croix et considérée comme le plus grand poète de l'Amérique coloniale'. Pratiquement tous les spécialistes ont confirmé cette attribution, non sans repérer quelques coquilles dues aux recopies successives. Notre traduction se fonde sur l'édition Tapia Méndez de 1993, Carta de Sor Juana a su confesor. Autodefensa

1 En français voir S.J.I. de la Cruz, Le Divin Narcisse précédé du Premier Songe et autres textes, Paris, Gallimard, 1987. Pour les villancicos (villanelles), voir le livret du disque K 627 Le Phénix de Mexico, Les chemins du baroque, 1999. Sor Juana a présents à l'esprit les modèles du Siècle d'Or espagnol : Góngora, Calderón, mais elle sait être originale, en particulier dans Le Songe, grand poème philosophique. D'autres poèmes religieux témoignent de l'attention qu'elle a portée aux Indiens, aux Noirs et aussi aux femmes; dans un poème à succès, elle rejette sur le premier sexe la réputation de libertinage. Si elle n'a guère la fibre mystique, elle s'intéresse beaucoup à la théologie ; ses villancicos ont un caractère catéchétique, voit Obras completas, Mexico, F.C.E., 4 vol. 1951-1957. 
espiritual, Monterrey, ed. Al Voleo El Troquel.

Le destinataire est le jésuite Antonio Núñez de Miranda, créole comme Sor Juana. Cet homme fort influent, austère et savant, grand prédicateur et expert de l'Inquisition, est le directeur spirituel de nombreuses religieuses. Ses biographes admettent qu'il avait une réputation d'autoritarisme spirituel. Confesseur de Sor Juana jeune fille, il l'a assurément poussée vers le couvent de Saint Jérôme où elle entre en 1668. Il semble que par la suite, il soit devenu plus exigeant ; la lettre que nous éditons consomma leur rupture, fait connu des spécialistes de Sor Juana mais non daté et attribué, jusqu'alors, au confesseur.

Juana est née vers 1648 à vingt lieues de Mexico, sans fortune ni noblesse et hors mariage - même si son père l'a reconnue avant de l'abandonner - mais elle est belle et très intelligente. Elle suit sa soeur aînée à l'école et sait lire à trois ans, feuilletant les livres en espagnol et en latin de son grand-père, puis une tante l'accueille dans la capitale. Une vice-reine pieuse et cultivée la prend à la cour où elle ne passe pas inaperçue. Que ce soit avant ou après son entrée au couvent, sa curiosité insatiable lui permet d'assimiler une culture certes beaucoup plus limitée que celle dont profite Anna Maria van Schurman, sa contemporaine, en Europe $^{2}$. Ainsi, la seule langue ancienne accessible est le latin. Comme elle le dira sans détour dans la Réponse à Sxeur Philothée de la Croix $x^{3}$, magnifique plaidoyer autobiographique de 1691 , publié en 1700 , elle choisit le couvent parce que c'est moins mauvais que le mariage pour ses chères études mais l'espoir de former des disciples y est nul. Heureusement, elle ne quitte pas vraiment le monde et s'adonne à la joie du travail intellectuel sous le patronage de Saint Jérôme et de Sainte Paule et ses filles.

Dans cette vice-royauté du Mexique colonial, les couvents, souvent riches, sont, avec leur musique religieuse, leur confiserie, leurs parloirs un élément considérable du prestige d'une ville. À intervalle régulier, la supérieure signe des dis-

2 «Lettre de l'humaniste Anna Maria Van Schurman (1607-1678) sur l'accès des femmes au savoir" commentée par Barbara Bulckaert, CLIO, Histoire, Femmes et Sociétes, 13, pp. 167-183.

3 Un évêque avait publié à son insu en 1690 sous le titre de Lettre athénagorique la critique qu'avait faite Sor Juana d'un sermon du portugais Antonio Vieira. Prenant le nom de Sœur Philotée, il lui conseillait d'abandonner ses activités profanes. On trouvera la traduction de la réponse dans Humanisme et religion chez Sor Juana Inés de la Cruz, Paris, Ed. Hispaniques/Publications de la Sorbonne, 1982, pp. 439-470. Autre traduction par le Prof. Nina Scott dans Women's studies, vol. 11, n 5, 1988, pp. 429438. 
penses du voeu de pauvreté. Les cellules sont des appartements que l'on peut acheter et il est bien vu de doter des pauvres «intéressantes», la splendeur du culte invitant à admettre des candidates sans dot si elles sont douées pour la musique. Le grand parloir est l'équivalent d'un salon où se répandent les nouvelles, où se polissent les moeurs des nouveaux riches. Grâce à Sor Juana, "la Dixième Muse», le couvent de St Jérôme attire l'élite intellectuelle locale. Le grand succès de l'Arc de triomphe que la religieuse a imaginé pour la «joyeuse entrée» du marquis de la Laguna lui assure, dès 1680 , le soutien de la nouvelle vice-reine. Elle a désormais une position sociale très forte et des correspondants de marque en Espagne et au Portugal ${ }^{4}$. Ce texte se situe dans ce climat. Tout porte à croire qu'une réelle amitié lia les deux femmes. Dans un registre conforme à la littérature courtisane de l'époque, Sor Juana a dédié de nombreux poèmes à la vice-reine. Après son retour en Espagne en 1688, celle-ci fit éditer les principaux écrits profanes et sacrés de son amie qui eurent alors un grand succès, $y$ compris auprès du clergé.

À Mexico même, ses amitiés haut placées et sa célébrité protègent la «Dixième Muse» pendant plusieurs années contre les menées possibles de ses adversaires au sein du clergé local. Mais vers 1693-1694, le changement est brutal. Sans explication, Sor Juana cesse de répondre aux lettres, vend beaucoup de livres au profit des pauvres, ferme son parloir et se réconcilie sans doute avec le vieux Père Núñez. Elle rédige alors quelques textes d'une dévote humilité mais elle ne reniera jamais ses écrits. Cette période mal connue a intrigué et divisé les historiens. Des travaux récents suggèrent que, devant le succès littéraire fracassant de la religieuse en Espagne, l'autorité ecclésiastique a mis fin à sa longue tolérances. En 1690 la Critique d'un sermon avait déjà scandalisé et suscité au moins un libelle anonyme. Mais un modus vivendi discret fut trouvé. Sor Juana s'est réfugiée dans ses études et ses livres tout en gardant la fonction de "contadora" (économe) du couvent et en mettant de réels talents de femme d'affaires au service d'une communauté où elle ne manquait pas de soutiens.

La Lettre au confesseur défend le droit à l'étude pour les femmes, un droit légitime puisque Dieu leur a donné l'intelligence qui est utile pour combattre la frivolité. D'ailleurs les exemples glorieux ne manquent pas dans le passé. En 1691,

4 Elle avait une relation privilégiée avec des religieuses nobles et cultivées de divers couvents portugais.

5 Il faut préciser que l'Inquisition est à l'époque très occupée par la sorcellerie, la bigamie et l'inconduite des prêtres. Elle n’a d'ailleurs brûlé personne à Mexico durant la vie adulte de Sor Juana. 
la Réponse à Saur Philothée reprend, développe ces arguments et en ajoute d'autres. Sor Juana récuse l'interprétation courante du "Mulieres in ecclesia taceant" de Saint Paul. Pour elle, étudier les sciences profanes permet de mieux comprendre la Bible. En fait, elle transforme le droit à l'étude en devoir d'étudier, en disciple de Sainte Paule. Le parallèle s'impose avec ses contemporaines, Anna Maria van Schurman et Gabrielle Suchon'. Elle va même plus loin, peutêtre en raison de son origine plus modeste, en réclamant de vraies écoles de filles avec des professeurs femmes. Ici, la parenté est évidente entre les deux lettres de Sor Juana, mais l'exigence d'autonomie morale apparaît beaucoup plus netrement dans cette lettre au confesseur.

Cette femme née pauvre au sein d'une société fermée sur son orthodoxie catholique allie l'érudition, le talent littéraire, l'autonomie morale, la conscience des problèmes et une forme de solidarité avec les autres femmes débouchant sur ce que l'on pourrait appeler, dans des termes contemporains, un programme revendicatif.

\section{Lettre de la mère Juana Inés de la Cruz à son confesseur, le R.P. Antonio Núñez de Miranda, de la Compagnie de Jésus}

\section{Pax Christi}

Depuis fort longtemps et de divers côtés, j'entends dire que, dans les conversations de Votre Révérence, la seule personne qui passe pour répréhensible, c'est moi, que votre acrimonie censure mes actions jusqu'à les traiter de scandale public ou autres qualifications non moins horribles. Ma conscience aurait pu m'inciter à me défendre car je ne suis pas entièrement propriétaire de mon crédit, solidaire que je suis d'une famille qui est la mienne et d'une communauté où je vis. Néanmoins, j'ai préféré sacrifier ma douleur à la vénération extrême et à l'affection filiale avec lesquelles, mon Père, je vous ai toujours respecté. Je préférais plutôt me voir mal jugée en toute chose que de laisser supposer, en polémiquant avec vous, que je passais les limites du respect que je vous dois. Je confesse en toute

6 Elle partage avec celle-ci la volonté d'une certaine autonomie face aux confesseurs.

Détail intéressant : Sor Juana invoque, comme Gabrielle Suchon, un texte peu connu de la Bible, le deuxième livre de Samuel ch. 14, qui relate le succès de la femme de Teqoa dans une ambassade particulièrement délicate, pour preuve de la capacité intellectuelle des femmes. Voir G. Suchon, Traité de la morale et de la politique, éd. S. Auffret, Paris, Des Femmes, 1988, et Du célibat volontaire, éd. ibid., Côté-femmes, 1994. 
naïveté que, ce faisant, je n'ai acquis aucun mérite auprès de Dieu car j'étais mue davantage par le respect tout humain envers votre personne que par la patience chrétienne. Je n'ignorais point la vénération et le crédit considérables dont, à juste titre, vous êtes l'objet, je savais que l'on vous écoute comme un oracle divin, que l'on estime vos paroles comme dictées par l'Esprit Saint et que, plus grande est votre autorité, plus grand est le préjudice que subit ma réputation. Cependant j'ai toujours résisté aux instances que, pour me pousser à répliquer, m'adressaient la raison ou bien, qui sait, l'amour-propre qui prend parfois le masque de la raison. Je jugeais que mon silence était le moyen le plus doux pour calmer votre courroux mais, le temps passant, j'ai dû reconnaître qu'à l'inverse, ma patience semble vous irriter, ce qui m'a déterminée à écrire cette réponse, mon Révérend Père, à la réserve de mon amour, de ma reconnaissance et de mon respect.

La cause, donc, de votre colère, mon Père très aimé, n'est autre que ces maudits poèmes que le ciel, à l'encontre de votre volonté, m'a donné le talent de composer. Pourtant j'ai tout fait pour échapper à cet office. J'ai tenté le plus possible de m'en dispenser, non que j'y trouvasse un motif de fierté ni de honte, car rimer a toujours été à mes yeux chose indifférente, et bien qu'il soit aisé d'énumérer combien de saints et de doctes ont pratiqué la poésie, je ne me mêlerai pas de la défendre car elle n'est ni mon père ni ma mère. Je dis seulement que je m'en abstenais pour complaire à votre Révérence sans chercher à comprendre la raison de son hostilité car le propre de l'amour, c'est d'obéir aveuglément. En outre cela s'accordait à la répugnance naturelle que j'ai toujours éprouvée à faire des vers, ce dont peuvent témoigner tous ceux qui me connaissent. Mais il ne m'a pas été possible d'observer cette loi avec tant de rigueur qu'elle ne souffrît aucune exception, en particulier deux villancicos en l'honneur de la Vierge Marie que j'ai écrits après des instances répétées et un silence de huit ans, avec votre permission, mon Révérend Père, car je la considérais alors comme plus nécessaire que celle de l'Archevêque-Vice-Roi mon supérieur. Et alors j'ai été si discrète que, la première fois j'ai refusé que l'on imprimât mon nom, et, la seconde, il fut mentionné à mon insu et contre mon avis. Les uns et les autres avaient été soumis à votre correction.

7 Villanelles. Ces poèmes à la fois religieux et populaires étaient chantés à l'église pour certaines fêtes, et renouvelés chaque année. Sor Juana en composa de nombreuses séries. Ceux qui sont mentionnés ici datent de 1676. L'archevêque fut en même temps vice-roi entre 1673 et 1680 . 
Ensuite vint l'Arc de triomphe de la cathédrale ${ }^{8}$ : la voilà ma faute impardonnable! Or j'ai d'abord été sollicitée à trois reprises et me suis récusée autant de fois jusqu'au jour où sont venus les deux directeurs de la fête qui, avant $\mathrm{d}$ 'avoir affaire à moi, s'adressèrent à la Mère Prieure, puis vinrent me trouver et m'ordonnèrent de m'en charger puisque le chapitre unanime avait voté en ce sens et que Monseigneur l'avait approuvé.

[Sor Juana explique ici pourquoi elle ne pouvait pas refuser et ajoute que la gratitude l'oblige parfois à prendre la plume pour des compliments mondains.]

Et maintenant, mon Père Vénéré, je supplie votre Révérence de laisser de côté un instant cette complaisance pour son propre jugement qui entraîne les hommes les plus saints ; dites-moi : puisque selon vous c'est un péché d'écrire des vers, en laquelle de ces occasions le mien a-t-il été si grave? Quand bien même ce serait un péché (et je ne sais pour quelle raison on le dirait tel) je serais excusée par les circonstances, par ces situations où je me suis trouvée bien contre mon gré, c'est l'évidence. Si à cette facilité que tout le monde me reconnaît se joignait une raison de vanité - sans doute est-ce plutôt de la mortification - pouvez-vous me souhaiter un châtiment plus dur que celui suscité par les applaudissements mêmes que vous déplorez? De quelle jalousie ne suis-je pas l'objet? Quelle action puis-je accomplir sans crainte? Quelle parole prononcer sans inquiétude? Les femmes se plaignent que je les surpasse, les hommes que je semble les égaler; les uns me voudraient moins de science, les autres disent que je n'en ai pas assez au regard de tant de succès. Les vieilles ne voudraient pas être dépassées en science, les jeunes que d'autres eussent bonne figure. Les uns et les autres voudraient que j'acceptasse les règles qu'ils édictent, et de tout cela résulte une sorte de martyre si étrange que je me demande si personne d'autre l'a jamais expérimenté.

Que puis-je encore faire valoir pour ma défense? Le seul fait de former mes lettres à peu près convenablement m'a valu une durable et pénible persécution, pour la simple raison que mon écriture avait l'air masculine et que ce n'était pas bienséant! On m'a donc obligée à la déformer exprès, toute la communauté m'en est témoin. Enfin j'aurais de quoi écrire non pas une lettre, mais plusieurs gros volumes. Et en quoi mes propos sont-ils coupables? Ai-je sollicité les applaudissements et les félicitations du public? Et les faveurs et les honneurs tout particu-

8 Lors de l'entrée solennelle d'un vice-roi, la Municipalité et l'Archevêché élevaient chacun un Arc éphémère décoré d'emblèmes à l'ancienne mode de la Renaissance. L'écrivain qui en fournissait le projet donnait aussi à l'impression un commentaire érudit, ingénieux... et flarteur. 
liers que m'accordent Leurs Excellences le Marquis et la Marquise par pure bienveillance et bonté sans égale, qu'ai-je fait pour les obtenir?

Il en alla bien différemment, et je peux apporter le témoignage de la Mère Jeanne de saint-Antoine, Prieure de ce couvent et personne que nul ne saurait soupçonner de mensonge. Lors de la première visite dont Leurs Excellences honorèrent cette maison, je lui demandai la permission de me retirer dans ma cellule sans les voir ni en être vue, comme si le Marquis et la Marquise m'eussent fait quelque tort! Ma seule raison était d'échapper à un succès qui se transforme en blessantes épines de persécution, et j'y serais parvenue si la Mère Prieure ne m'avait commandé le contraire. En quoi est-ce ma faute si j'ai plu à Leurs Excellences? Je ne le méritais pas, mais aurais-je pu opposer un refus à des personnes toutes puissantes? Devrai-je me désoler qu'elles m'honorent de leurs visites? Vous savez parfaitement, mon Père, qu'il n'en est rien.

[Sor Juana fait remarquer qu'elle n'est pas responsable des incursions de la Cour dans les couvents de femmes, y compris dans les quelques monastères très observants comme le Carmel.]

Mon étude n'a causé tort ni préjudice à personne, d'autant moins que je m'y suis livrée si privément que je n'ai même pas eu recours à la direction d'un maitre; je me suis contentée de faire au mieux avec moi-même et mon travail. Je n'ignore pas que fréquenter publiquement les écoles serait malséant pour une femme honnête en raison de l'inévitable familiarité avec des hommes et que tel doit être le motif qui [leur] interdit l'accès aux écoles. Et si l'on ne leur y prévoit pas de place, ce sera parce que l'État n'a pas besoin d'elles pour les charges et les magistratures (dont elles se voient exclues pour le même motif de décence) et qu'il néglige ce qui leur est inutile. Mais les études privées et particulières, qui les a interdites aux femmes? N'ont-elles pas une âme rationnelle comme les hommes? Pourquoi celle-ci ne jouirait-elle pas aussi de ce privilège qu'est la lumière de la science? L'âme d'une femme n'est-elle pas apte à recevoir autant de grâce et de gloire de Dieu que la leur? Pourquoi ne serait-elle pas apte à recevoir autant de connaissance et de science qui sont choses moins nobles? Quelle révélation divine, quelle décision de l'Église, quelle loi de la raison a fait pour nous une loi si sévère?

La science serait-elle un obstacle et non une aide pour faire son salut? Saint Augustin, saint Ambroise et tous les autres saints docteurs ne sont-ils pas sauvés? Et vous, mon Révérend Père, qui êtes si érudit, ne pensez-vous pas vous sauver? Et si vous me répondez que chez les hommes le problème est différent, voici ma réponse: sainte Catherine, sainte Gertrude n'ont-elles pas étudié? Et ma Mère 
sainte Paule, tout en atteignant une haute contemplation et en réalisant de difficiles fondations, n'a-t-elle pas étudié même le grec? N'a-t-elle pas appris l'hébreu? Élève de mon Père saint Jérôme, n'a-t-elle pas su comprendre et pénétrer les Écritures, comme le saint l'avoue lui-même? Ne célèbre-t-il pas dans l'une de ses épîtres Blésille, fille de Paule, docte en toute sorte d'études dès l'âge le plus tendre puisqu'elle mourut à vingt ans?

Pourquoi donc ce qui est bon chez toutes va-t-il être mauvais pour moi? Suisje la seule pour qui les livres soient un obstacle au salut? Si j'ai lu les poètes' et les orateurs profanes, faiblesse que je partage avec saint Jérôme lui-même, je lis aussi les Docteurs de l'Église et la Sainte Écriture, et je ne puis nier que je dois aux premiers des bienfaits innombrables et de bonnes règles de vie. En effet, quel chrétien n'a pas honte de ses colères devant la patience du païen Socrate? Qui pourra être ambitieux devant la modestie de Diogène, le cynique. Qui ne rend grâce à Dieu pour l'intelligence d'Aristote? Enfin quel catholique n'est pas confondu s'il contemple la somme de vertus morales de tous les philosophes païens?

Suis-je coupable si le moment que j'aurais pu passer au parloir à dire des sottises, ou dans ma cellule à médire de tout ce qui se passe hors du couvent ou dedans, ou bien à me disputer avec une soeur, ou à gronder une pauvre servante, ou à divaguer par la pensée dans le monde entier, si ce moment, dis-je, je le passe à étudier. En outre, c'est Dieu qui m'a donné cette inclination, et cela ne m'a pas paru contraire à sa loi très sainte ni aux obligations de mon état. Tel est mon caractère. Je [ne] me suis [pas $]^{10}$ faite moi-même. Telle je suis née, telle je mourrai. Vous voulez, mon Père, qu'en me faisant violence je me sauve ignorante, mais, Bien-aimé Père, cela est-il impossible avec la science? C'est quand même pour moi un chemin plus doux. Pourquoi devoir faire son salut par la voie de l'ignorance si cela répugne à votre nature? La Bonté Infinie de Dieu n'inclut-elle la Sagesse et la Science parfaites? Pourquoi donc l'ignorance devrait-elle lui être plus agréable que la connaissance? Que saint Antoine fasse son salut avec sa sainte ignorance, bravo, mais saint Augustin a choisi une autre voie et aucun des deux ne fait fausse route.

Pourquoi donc ce déplaisir de votre Révérence? Pourquoi dites-vous que si vous aviez su que je me mettrais à écrire des vers, vous m'auriez mariée au lieu de me mettre au couvent? Eh bien, Père très aimant (que je contredis contrainte et

9 Le manuscrit dit "profetas», coquille probable déjà corrigée sur notre source.

10 La négation n'est pas dans le manuscrit mais elle convient au sens général du paragraphe. Ces crochets figurent déjà dans notre source. 
forcée, pleine de honte, en des termes qui n'auraient pas dû franchir mes lèvres), quelle autorité directe avait votre Révérence pour disposer de ma personne et du libre-arbitre que Dieu m'a concédé? Vous n'avez que celle que mon affection vous a donnée et vous donnera toujours.

[Sor Juana note qu'elle a rę̧u sa dot d'une tierce personne, que son unique dette envers le Père Núñez, consiste en quelques leçons (de latin sans doute) payées par lui.]

Serais-je donc hérétique? Et si je l'étais, devrais-je parvenir de force à la sainteté? Plût à Dieu qu'il fût possible de devenir sainte sur commande! J'aurais été sûre de le devenir. Mais je pense qu'on le devient par la persuasion, non par l'autorité, et en fait d'autorité, j'ai eu des supérieurs pour s'en charger. $\mathrm{Si}^{11}$ elles sont modérées et prudentes, les règles et pressions extérieures nous rendent sages et modestes, mais si elles sont excessives, elles nous désespèrent. Quant à la sainteté, seuls la grâce et le secours de Dieu peuvent la faire naître. Sur quoi se fonde votre courroux? Pourquoi vouloir m'ôter mon crédit? Pourquoi me faire passer pour scandaleuse aux yeux de tous? Vous ai-je fatigué, mon Père, de quelque requête? Vous ai-je demandé quelque secours dans mes nécessités? Ai-je été fâcheuse en matière spirituelle ou temporelle? Vous revient-il de me corriger pour quelque raison d'obligation, de parenté, de tutorat, d'autorité canonique ou quelqu'autre? Si votre mobile, mon Révérend Père, est pure charité, qu'elle ressemble à de la charité et qu'elle procède comme telle avec douceur, car m'exaspérer n'est pas le moyen de me réduire et ma nature n'est pas servile au point d'accomplir sous la menace ce que ne me persuade pas la raison, ni pour des considérations humaines ce que je ne ferais pas pour Dieu. Me priver de tout ce qui, dans les bornes du licite, m'est agréable, il convient que je le fasse à titre de mortification, lorsque moi-même je déciderai de faire pénitence, non parce que votre Révérence aura eu la volonté de l'obtenir à force de répréhensions, lesquelles ne me sont pas adressées en secret comme le stipule la correction paternelle (puisque vous voulez vous considérer comme mon père ce qui est pour moi un grand bonheur), mais publiquement, devant tout le monde, sujettes aux interprétations et commérages de chacun.

N'est-il pas inévitable, Mon Père, que cela me fasse de la peine venant d'une personne que j'aime avec tant de vénération et que je vénère et estime avec tant d'amour. Si vos répréhensions s'appliquaient à un écrit scandaleux, ma docilité est telle que, bien que ni en matière spirituelle ni en matière temporelle je ne dépende de votre Révérence, je m'en éloignerais et je tenterais de m'amender, et

11 Nous supprimons ici un «mais» ("pero") qui n'a guère sa place. 
de vous satisfaire, même contre ma propre pente. Mais s'il ne s'agit que de divergence d'opinion, puisqu'en substance, faire des vers ou n'en point faire est indifférent, et que je les abhorre au point que les écrire est sans cesse pour moi la pire des pénitences, pourquoi me tourmenter à ce point?

[Non sans quelque cruauté, Sor Juana fait sentir ici à son confesseur qu'elle n'est guère sensible à son "charisme»; elle lui dit que d'autres prêtres peuvent remplir ce rôle, que «le ciel a beaucoup de clés» et ajoute que c'est la sincérité qui la pousse à cette pénible démarche en demandant pardon pour le cas où elle se serait exprimée de façon blessante ou peu respectueuse...]

Ma seule intention est de vous supplier, mon Père, s'il ne vous plaît pas de m'accorder votre faveur, de ne plus vous souvenir de moi, si ce n'est pour me recommander à Dieu dans vos prières. Je suis sûre qu'en raison de votre grande charité, vous le ferez véritablement.

Je demande à Dieu qu'il me garde votre Révérence comme je le souhaire.

Bien à vous.

En ce couvent de Saint Jérôme de Mexico. Juana Inés de la Cruz 